\title{
A conditional value-at-risk model for insurance products with a guarantee
}

\section{Andrea Consiglio* and Antonio Pecorella}

University of Palermo, Palermo, Italy

Email: consiglio@unipa.it Email: tonpec@unipa.it

*Corresponding author

\section{Stavros A. Zenios}

University of Cyprus, Nicosia, Cyprus

Email: zenioss@ucy.ac.cy

\begin{abstract}
We propose a model to select the optimal portfolio which underlies insurance policies with a guarantee. The objective function is defined in order to minimise the conditional value-at-risk (CVaR) of the distribution of the losses with respect to a target return. We add operational and regulatory constraints to make the model as flexible as possible when used for real applications. We show that the integration of the asset and liability side yields superior performances with respect to naive fixed-mix portfolios and asset based strategies. We validate the model on out-of-sample scenarios and provide insights on policy design.
\end{abstract}

Keywords: asset-liability management; conditional value-at-risk; CVaR; policies with a minimum guarantee; portfolio selection.

Reference to this paper should be made as follows: Consiglio, A., Pecorella, A. and Zenios, S.A. (2009) 'A conditional value-at-risk model for insurance products with a guarantee', Int. J. Risk Assessment and Management, Vol. 11, Nos. 1/2, pp.122-137.

Biographical notes: Andrea Consiglio is professor of mathematical finance at the University of Palermo, Italy. He held positions at the University of Calabria and at the University of Cyprus. He has participated in consultancy projects with the Banca della Svizzera Italiana, Switzerland and Prometeia, Italy. He has co-authored one book and numerous articles for various leading academic journals. In 2006 he was awarded the EURO Excellence in Practice Award, jointly with Stavros A. Zenios and Flavio Cocco. His research interests encompass many areas in the field of financial modelling and computational finance. He holds a $\mathrm{PhD}$ in applied mathematics to finance and economics.

Antonio Pecorella is associate professor of mathematics at the University of Palermo, Italy. The focus of his research is on mathematical models for finance and economics.

Stavros A. Zenios holds a BSc in Mathematics from London University, a BEng in Electrical Engineering from Council of Engineering Institutions, MA, USA, a PhD in Engineering-Management Systems from Princeton University and an Honorary MA from the University of Pennsylvania. Prior to joining the University of Cyprus to serve as the first Dean of the School of Economics 
and Management he was a tenured faculty member of the Wharton School, University of Pennsylvania. He also held visiting academic appointments at the Sloan School of the Massachusetts Institute of Technology (MIT), University of Haifa (Israel), University of Vienna and the Universities of Bergamo, Milano and Urbino. He is known internationally for his work in computational finance and financial services, high-performance and parallel computations and operations research. He has authored two books, edited twelve books and journal issues and co-authored over 130 scholarly articles. He holds two US patents on financial engineering methods.

\section{Introduction}

In the last years we have witnessed wild fluctuations of the financial markets due to external events (Twin Towers effect, Iraq war) and internal events (speculative bubbles, bankruptcies in the US and European market). Many investors, frightened by these unforeseen facts, have withdrawn their capitals from the perils of naked positions and moved towards more protected investments.

The need for safe investments has seen the flourish of financial products which promise a baseline minimum return in the case of negative performances of the underline assets and, at the same time, allow the investor to participate in the returns of a portfolio with high equity content.

Such products are inherited from the insurance industry where policies with a minimum guarantee have gained the favour of householders interested not only in health insurance.

However, a minimum guarantee product must be handled carefully. As seen in the case 'Equitable Life vs Hyman', the insurance company had to close its funds after suffering substantial losses due to a decision of the House of Lords in interpreting, negatively, the discretion with which Equitable had structured the bonus to the policyholders.

The literature in this field has, mainly, focused on pricing the minimum guarantee option which is embedded in the policy contract (Bacinello, 2001, 2003; Grosen and Jørgensen, 2000; Miltersen and Persson, 1999). Giraldi et al. (2003) investigate the same aspect but put more emphasis on the operational constraints faced by the insurance company when pricing such instruments.

An integrative approach was laid for the first time by Carinõ and Ziemba (1998) in their model developed for the Japanese insurance firm Yasuda Kasai. This new strand of literature has been followed by other examples (Consigli and Dempster, 1998; Høyland, 1998; Mulvey and Thorlacius, 1998) which, basically, aim at a global formulation of the asset and liability structure of a firm and try to maximise some criteria in order to determine the best mix of decision variables. The models obtained are quite flexible and easily manage operational constraints, such as institutional restrictions, transaction costs and other limitations on the asset and liability composition.

However, a global formulation suffers a drawback of empirical content when we observe that insurance products are equipped with hidden options which make their behaviour highly nonlinear. For example, a special feature of minimum guarantee policies is that the liability is lifted every time a bonus is paid and the minimum guarantee applies to the increased liability: what is given cannot be taken away. 
Recently, Consiglio et al. (2001, 2007) proposed a model for the Italian insurance policies which attempt to conjugate the power of asset and liability management and the need for taking into account the effects of minimum guarantee bonuses. The same approach is proposed in Consiglio et al. $(2003,2006)$ where the model is extended to a specific class of insurance product with a bonus mechanism adopted in the UK.

One of the issues which motivates this paper is the introduction of an objective function which relies on more realistic assumptions. Behavioural studies in finance have yielded evidence that investors suffer from so-called myopic risk aversion. In other words, investors penalise more negative deviations from a given target than positive ones (Kahneman and Tversky, 1979). A portfolio model which weighs, differently, upside from downside deviations is in Consiglio and Zenios (2001). Further developments can be found in Dembo and Mausser (2000) where they determine a put-call objective function with strike price given by the benchmark return. Lately, greater interest has been devoted to the optimisation of conditional value-at-risk (CVaR). According to the axioms given in Artzner et al. (1999), CVaR is a coherent measure of risk and Uryasev and Rockafellar (2000) show that, under certain mild hypotheses, minimisation of $\mathrm{CVaR}$ can be handled through algorithms to optimise smooth functions.

In Consiglio et al. $(2001,2007)$ regulatory and solvency constraints are not embedded in the model and they are checked in a post-optimality phase. The reason is mainly technical. Explicit inequalities add nonlinear constraints to the programming model and their number grows with the number of scenarios used to deal with uncertainty. In this paper we will introduce such constraints directly in the programming model and show their effect on the performance of the policy.

The paper is organised as follows: in Section 2 we define the dynamic equations which describe the base model, the operational constraints imposed by regulators and by solvency requirements and a CVaR objective function. In Section 3 we report the mathematical programming model and Section 4 analyses the features of the model and discusses the results obtained with multipurpose optimisation packages.

\section{Modelling issues}

The base model is made up of a set of constraints which define the dynamics of three accounts:

- a liability account that grows according to the contractual guaranteed rate and bonus provision

- an asset account that grows according to the portfolio returns, net any payments due to death or policy surrenders

- an equity account that keeps track of the funds needed to cover shortfalls of the portfolio return against the guarantee.

These three accounts are time dependent and evolve over a given time horizon. The uncertainty of the parameters which enter the model is captured by means of a set of discrete scenarios. All portfolio decisions are made at $t=0$. 
The multi-period dynamics of these accounts are conditioned on discrete scenarios of realised asset returns and the composition of the asset portfolio. At maturity, the difference between the asset and the liability accounts is the surplus realised by the firm after it has fulfilled its contractual obligations. In the policies considered here, this surplus remains with the shareholders. This surplus is a random variable and the minimisation of the CVaR of an appropriate loss function is an eligible target for the managers of the policy.

\subsection{Notation}

We let $\Omega$ denote the index set of scenarios $l=1,2, \ldots N, \mathcal{U}$ the universe of available asset instruments and $t=1,2, \ldots, T$, discrete points in time from today $(t=0)$ until maturity $T$. The data of the problem are as follows:

- $r_{i t}^{l}$, rate of return of asset $i$ during the period $t-1$ to $t$ in scenario $l$.

- $r_{f t}^{l}$, risk free rate during the period $t-1$ to $t$ in scenario $l$

- $g$, minimum guaranteed rate of return

- $\alpha$, participation rate indicating the percentage of portfolio return paid to policyholders

- $\rho$, regulatory equity to debt ratio

- $\Lambda_{t}^{l}$, probability of abandonment of the policy due to lapse or death at period $t$ in scenario $l$.

The variables of the model are defined as follows:

- $x_{i}$, percentage of initial capital invested in the $i$ th asset

- $y_{A t}^{l}$, expenses due to lapse or death at time $t$ in scenario $l$

- $z_{t}^{l}$, shortfall below the guaranteed rate at time $t$ in scenario $l$

- $A_{t}^{l}$, asset value at time $t$ in scenario $l$

- $E_{t}^{l}$, total equity at time $t$ in scenario $l$

- $L_{t}^{l}$, liability value at time $t$ in scenario $l$

- $R_{P t}^{l}$, portfolio rate of return during the period $t-1$ to $t$ in scenario $l$

- $y_{t}^{+l}$, excess return over $g$ at time $t$ in scenario $l$

- $y_{t}^{-l}$, shortfall return under $g$ at time $t$ in scenario $l$.

\subsection{Definitional constraints}

We invest the premium collected $\left(L_{0}\right)$ and the equity required by the regulators $\left(E_{0}=\rho L_{0}\right)$ in the asset portfolio. Our initial endowment $A_{0}=L_{0}(1+\rho)$ is allocated to assets in proportion $x_{i}$ such that $\sum_{i \in \mathcal{U}} x_{i}=1$ and the dynamics of the portfolio return is given by

$$
R_{P t}^{l}=\sum_{i \in \mathcal{U}} x_{i} r_{i t}^{l}, \text { for } t=1,2, \ldots T, \text { and for all } l \in \Omega .
$$


The investment variables are non-negative so that short sales are not allowed.

At each point in time and for each scenario the return of the policy is given by

$$
\max \left[\alpha R_{P t}^{l}, g\right], \text { for } t=1,2, \ldots T, \text { and for all } l \in \Omega,
$$

Note that the max operator introduces a discontinuity in the model. To circumvent this difficulty we introduce variables $y_{t}^{+l}$ and $y_{t}^{-l}$ to measure the portfolio excess return over the guaranteed rate and the shortfall below the guarantee, respectively. They satisfy

$$
\begin{gathered}
\alpha R_{P t}^{l}-g=y_{t}^{+l}-y_{t}^{-l}, \text { for } t=1,2, \ldots T, \text { and for all } l \in \Omega, \\
y_{t}^{+l} \geq 0, y_{t}^{-l} \geq 0, y_{t}^{+l} y_{t}^{-l}=0, \text { for } t=1,2, \ldots T, \text { and for all } l \in \Omega .
\end{gathered}
$$

\subsubsection{Liability constraint}

Liabilities will grow at a rate which is at least equal to the guarantee. Excess returns over $g$ are returned to the policyholders according to the participation rate $\alpha$. The dynamics of the liability account are given by

$$
L_{t}^{l}=\left(1-\Lambda_{t}^{l}\right) L_{t-1}^{l}\left(1+g+y_{t}^{+l}\right), \text { for } t=1,2, \ldots T, \text { and for all } l \in \Omega .
$$

Any excess return, $y_{t}^{+l}$, is added to the liabilities and the guarantee applies to the lifted liabilities.

\subsubsection{Equity constraint}

In the base model shortfalls are funded through equity. We assume that equity is reinvested at the risk-free rate and is returned to the shareholders at the end of the planning horizon. The dynamics of the equity are given by

$$
E_{t}^{l}=E_{t-1}^{l}\left(1+r_{f t}^{l}\right)+z_{t}^{l}, \text { for } t=1,2, \ldots T, \text { and for all } l \in \Omega .
$$

At maturity the shareholders will get the equity reinvested at the risk free rate plus any dividend left after the liability is paid to the policyholders. The final equity, $E_{T}^{l}$, is the lower bound reward for the shareholders. The optimal choice of the initial portfolio must yield a final dividend, $A_{T}^{l}-L_{T}^{l}$, greater than the final equity. On the contrary, the equity investment would yield a rate which is less or equal to the risk free. Such portfolios are penalised by the optimisation process.

\subsubsection{Asset constraint}

The asset dynamics have to take into account the cash infusion that funds shortfalls, $z_{t}^{l}$ and the outflows due to actuarial events $y_{A t}^{l}$. In particular, at each period the insurance company makes payments due to policyholders abandoning their policies because of death or lapse. Payments are equal to the value of the liability times the probability of abandonment, i.e.

$$
y_{A t}^{l}=\Lambda_{t}^{l} L_{t-1}^{l}(1+g+), \text { for } t=1,2, \ldots T, \text { and for all } l \in \Omega .
$$

Whenever the portfolio return is below the guaranteed rate we need to infuse cash into the asset portfolio in order to meet the final liabilities. The shortfall account is modelled by the dynamics 


$$
z_{t}^{l}=y_{t}^{-l} L_{t-1}^{l}, \text { for } t=1,2, \ldots T, \text { for all } l \in \Omega .
$$

The asset dynamics are, therefore, given by

$$
A_{t}^{l}=A_{t-1}^{l}\left(1+R_{P t}^{l}\right)+z_{t}^{l}-y_{A t}^{l}, \text { for } t=1,2, \ldots T, \text { and for all } l \in \Omega .
$$

\subsection{Operational constraints}

We introduce operational constraints in order to incorporate technical considerations about asset allocation, equity infusion, limitations and requirements of the regulators on such products into the model. Limitations on the asset allocation can easily be added imposing box constraints on the decision variables $x_{i}$

$$
x_{i}^{L} \leq x_{i} \leq x_{i}^{U}, \text { for all } i \in \mathcal{U} .
$$

Note that short sales are not allowed, which implicitly means that $x_{i}^{L}=0$. Box constraints are easy to handle and do not imply any technical problem for the solution algorithm.

In Consiglio et al. (2001) the equity infused is not bounded from the above, although the optimisation model will penalise asset allocations which yield high equity values. An upper bound for the equity level is usually required by the managers to be sure that the optimal policy is not too demanding for shareholders and, thus, rejected as unrealistic. Such constraints are simply given by

$$
E_{t}^{l} \leq E^{U}, \text { for } t=1,2, \ldots T, \text { and for all } l \in \Omega .
$$

The regulatory board compels insurance companies to maintain a certain ratio between equity and liability for all the life of the specific product. It is, therefore, necessary that

$$
\frac{E_{t}^{l}}{L_{t}^{l}} \geq \rho, \text { for } t=1,2, \ldots T, \text { and for all } l \in \Omega .
$$

Note that at time $t=0$ the amount of equity is fixed to the minimum requirements, i.e. $E_{0}=\rho L_{0}$.

In Consiglio et al. (2001), Equations (11) and (12) are not explicitly introduced but only checked in a post-optimality phase. Keeping the asset allocation fixed, they deal with the probability of insolvency through a simulation analysis which determines the choices of the debt structure (the combination of additional equity and short or long debt) and charges to the policyholders. This approach is sub-optimal but it has the advantage of saving the mathematical structure of the programming model.

In the current formulation, Equations (11) and (12) must be satisfied at each time step $t=1,2, \ldots, T$, thus requiring equity and liability levels in each period. From a technical point of view, this turns to adding a set of nonlinear constraints for each period and for each scenario. Although such nonlinearities do not have a complex mathematical form per se, the 'curse of dimensionality' arises when we consider a realistic number of scenarios and time steps. With hundreds of scenarios and a five-ten year horizon, the nonlinearly constrained programming model becomes unstable and tailored algorithms are needed to determine the optimal solution (which, however, is not guaranteed to be a global optimum). 


\subsection{Objective function}

In this paper we will prioritise the objective of the shareholders who aim to improve the performance of their investments. Alternative views can be considered, as in Consiglio et al. (2006). The performance measure will rely on quantities whose realisation is scenario dependant and mature at the end of the horizon. In detail, at the end of the planning period the value of the assets is worth $A_{T}^{l}$ under each scenario. With this amount the insurance company has to liquidate its liability towards the policyholders $\left(L_{T}^{l}\right)$ and refund the shareholders of the capital $\left(E_{T}^{l}\right)$ infused to cover shortfalls under the minimum guarantee. Note that $E_{T}^{l}$ is evaluated at the risk free rate (Equation (6)). The reward of the shareholders under each scenario is, thus, given by

$$
W_{T}^{l}=A_{T}^{l}-L_{T}^{l}-E_{T}^{l} .
$$

The return on equity under each scenario $\left(\mathrm{ROE}^{l}\right)$ must be calculated not only on the upfront investment $\left(E_{0}=\rho L_{0}\right)$ but on the whole equity infused, so we have

$$
\operatorname{ROE}^{l}=\frac{W_{T}^{l}}{E_{T}^{l}}=\frac{A_{T}^{l}-L_{T}^{l}-E_{T}^{l}}{E_{T}^{l}} .
$$

In terms of excess ROE, we can write

$$
\theta^{l}=\frac{A_{T}^{l}-L_{T}^{l}}{E_{T}^{l}}-1 .
$$

Managers, announcing their strategies, usually illustrate their future achievements in terms of annual target returns. Their performance is, thus, measured with respect to the deviations from this target. In turn, shareholders will make and review their decisions on keeping or dropping quotas of the company according to the adherence of the current performance to the announced target.

In selecting the portfolio, it is then opportune to choose those asset allocations which guarantee a minimum deviation from the target. Since $\theta^{l}$ is scenario dependant, such a decision must take into account the probability distribution of the excess ROE.

In Consiglio et al. (2001), the objective function maximises the certainty equivalent of the utility of the excess ROE. The utility function is assumed to be logarithmic. The choice of an appropriate utility function is a controversial issue and, in many cases, this aspect is left open to an array of options (Consiglio et al., 2007).

On the contrary, target based performance measures are much more intuitive and allow easier interpretations from the managers. If we denote the target excess ROE with $\Theta$, we can define with

$$
D^{l}=\Theta-\theta^{l}
$$

the loss with respect to the given target for each scenario $l \in \Omega$. We can imagine $D^{l}$ as a realisation of a sample drawn from the probability distribution of the losses. Each $D^{l}$ depends on the portfolio selected at time $t=0$ and an eligible objective for the managers would be that of minimising the conditional VaR of the losses' sample distribution. 
Similar objective functions can be found in Consiglio and Zenios (2001), where a mean absolute deviation model with infinity penalty on negative downsides is used to track the index of international government bonds or, in Dembo and Rosen (1999), where they define the regret as the portfolio under-performance with respect to a fixed target and use its expectation for portfolio selection models. An interesting analysis on the relationship between CVaR and regret models can be found in Testuri and Uryasev (2003). They showed, also numerically, that both approaches yield the same portfolios for suitable values of the target and confidence level.

Given the loss function $D^{l}$, the CVaR of the sample distribution is approximated by

$$
\xi(1-\beta)+\frac{1}{N} \sum_{l=1}^{N}\left[D^{l}-\xi\right]^{+},
$$

where $\beta$ is the confidence level, $\xi$ is the VaR, and $[\cdot]^{+}$denotes the positive part of its operand.

\section{The mathematical programming model}

We can now assemble the set of equations defined in the previous section and, after trivial substitutions, we obtain the following mathematical programming model:

$$
\text { Minimise } \xi(1-\beta)+\frac{1}{N} \sum_{l=1}^{N} S^{l}
$$

s.t.

$$
\begin{gathered}
\sum_{i \in \mathcal{U}} x_{i}=1, \\
\alpha \sum_{i \in \mathcal{U}} x_{i} r_{i t}^{l}=g+y_{t}^{+l}-y_{t}^{-l}, \text { for } t=1,2, \ldots T, \text { and for all } l \in \Omega \\
L_{t}^{l}=\left(1-\Lambda_{t}^{l}\right) L_{t-1}^{l}\left(1+g+y_{t}^{+l}\right), \text { for } t=1,2, \ldots T, \text { and for all } l \in \Omega \\
E_{t}^{l}=E_{t-1}^{l}\left(1+r_{f t}^{l}\right)+y_{t}^{-l} L_{t-1}^{l}, \text { for } t=1,2, \ldots T, \text { and for all } l \in \Omega \\
A_{t}^{l}=A_{t-1}^{l}\left(1+\sum_{i \in \mathcal{U}} x_{i} r_{i t}^{l}\right)+L_{t-1}^{l}\left[y_{t}^{-l}\left(1+g+y_{t}^{+l}\right)\right], \\
\text { for } t=1,2, \ldots T, \text { and for all } l \in \Omega \\
\rho L_{t}^{l} \leq E_{t}^{l} \leq E^{U}, \text { for } t=1,2, \ldots T, \text { and for all } l \in \Omega \\
S^{l} \geq \Theta-\frac{A_{T}^{l}-L_{T}^{l}}{E_{T}^{l}}-\xi+1, S^{l} \geq 0, \text { for all } l \in \Omega \\
y_{t}^{+l}, y_{t}^{-l} \geq 0, \text { for } t=1,2, \ldots T, \text { and for all } l \in \Omega \\
x_{i}^{L} \leq x_{i} \leq x_{i}^{U} \text { for all } i \in \mathcal{U} .
\end{gathered}
$$


We outline here some key points of Equations (18)-(27):

- The original formulation of the objective function (Equation (17)) has been modified by adding the set of constraints in Equation (25) and by carrying the minimisation over the sum of the positive dummy variables $S^{l}$. We operate in this way in order to avoid the discontinuity of the first derivative of the term $\left[D^{l}-\xi\right]^{+}$. Although this implies the increment of the number of nonlinear inequalities, smooth nonlinear models are easier to handle, especially when the dimensionality plays a crucial role in the convergence of the solution process.

- We condensed in the set of inequalities in Equation (24), the regulatory requirements and the operational constraints on the equity. Note that caution must be taken in setting the upper bound of the equity variables. In fact, since the lower bound cannot be determined a priori because it depends on the endogenous variables $L_{t}^{l}$, we run the risk of unfeasible problems when it happens that $\rho L_{t}^{l} \geq E^{U}$. To this purpose, we first set the upper bound $E^{U}$ to a large value and then try to tighten it gradually until an unfeasible problem is encountered.

- The number of variables and constraints of Equations (18)-(27) depends on the number of scenarios drawn from the multivariate distribution of the asset classes returns. In particular, we have that the number of variables amounts to $m=|\mathcal{U}|+6 \cdot N+5 \cdot T$, while the number of general constraints (not including bounds on the variables) amounts to $n=7 \cdot N+6 \cdot T+1$. We obtain that, for a number of scenarios ranging from $100-500, m+n$ ranges from $1200-7000$, thus classifying the model as large scale.

\section{Model analysis}

The aim of this section is to show that the model proposed can assist managers in designing lines of business which appear to be very complex when seen from an integrative perspective.

In this respect, simulation models which synthesise the dynamics of asset, liability and equity are of common use in the insurance industry (Giraldi et al., 2003; Siglienti, 2000). In our formulation, we take a step further by letting the strategic choice be an endogenous variable of the model, so that simulation and optimisation are coalesced in a unique phase.

The asset classes considered in our study are 23 stock indices of the Milano Stock Exchange and three Salomon Brother indices of the Italian Government bonds. We employ a simple approach for generating scenarios using only the available data without any mathematical modelling, by bootstrapping a set of historical records. Each scenario is a sample of returns of the assets obtained by sampling returns that were observed in the past. Dates from the available historical records are selected randomly and, for each date in the sample, we read the returns of all asset classes realised during the previous month. These samples are scenarios of monthly returns. To generate scenarios of returns for a long horizon - say ten years - we sample 120 monthly returns from different points in time. The compounded return of the sampled series is one scenario of the ten-year return. The process is repeated to generate the desired number of scenarios for the ten-year period. With this approach, 
the correlations among asset classes are preserved. In order to take account of the serial dependence of the returns, we use a block sampling scheme where each block has length of 12 months (Efron and Tibshirani, 1998).

Additional scenarios could also be included, although methods for generating them should be specified. Model-based scenario generation methods for asset returns are popular in the insurance industry - e.g. Wilkie's model (Wilkie, 1995) - and could be readily incorporated into the scenario optimisation model. Alternatively, one could use expert opinion or 'scenario proxies' as discussed in Dembo et al. (2000).

In general, a large number of scenarios is needed in order to get a reliable representation of the probability distribution of the underlying financial factors. However, the larger the number of scenarios, the more likely it is that the model incurs numerical instability and delivers an unfeasible solution. For practical applications, variance reduction and importance sampling methods can significantly lessen the number of instances to be sampled. In our experiments, we run the model over sets of scenarios with size 500, 1000 and 2000. The model was implemented in the algebraic modelling language GAMS of Brooke et al. (1992) using the solvers SNOPT and CONOPT.

The scenarios were bootstrapped from monthly records of the ten year period starting in January 1993 and ending in February 2004. The monthly returns are compounded to yearly returns. The probability that a policyholder abandons the policy is $\operatorname{Prob}($ death $)+\operatorname{Prob}($ lapse). In our experiments we set lapse probabilities to zero and use death probabilities from the Italian mortality tables.

We consider an initial liability $L_{0}=1$ for a contract with participation rate $\alpha=85 \%$. The model is tested for guarantee rates $g$ and equity to liability ratios $\rho$ ranging from $1-10 \%$.

A deeper analysis of a similar model can be found in Consiglio et al. (2008). In this paper, we focus our attention on out-of-sample tests in order to assess

- the gain in making a choice which takes into account assets and liabilities

- the impact of the different constraints added.

To this purpose we split the sample data into two parts. The first part includes data from January 1993 to January 2001 and it will be used to select the optimal asset allocation. The second part includes data from February 2001 until February 2004. From the latter, we bootstrap a wide sample of scenarios and simulate the optimal portfolios. Note that the out-of-sample scenarios include the Twin Tower shock, while the portfolio is optimised on a sample of scenarios previous to this catastrophic event.

\subsection{The effect of integration}

To test the effect of integration, we first solve the model in Equations (18)-(27) for $g=3 \% \rho=4 \%$ and $\Theta=1.6289$ which corresponds to $5 \%$ per year. We simulate the portfolio over a set of 5000 scenarios, $\Omega^{*}$, drawn from the out-of-sample dataset. For each scenario, the dynamics of the asset, liability and equity is used in order to evaluate $A_{T}^{l}, L_{T}^{l}$ and $E_{T}^{l}$. We then calculate the returns on equity $\mathrm{ROE}^{l}$ and losses $D^{l}$ for each $l \in \Omega^{*}$. Finally, we order the losses and compute the VaR at $5 \%$ and the expected ROE. 
When sophisticated mathematical models are used, it is customary to question whether a more naive approach could perform equally well. We, thus, compare the performance of the optimal portfolio with a set of 100 portfolios randomly drawn from the simplex $\sum_{i \in \mathcal{U}} x_{i}=1$.

To assess the effect of integrating the asset and liability dynamics in the decision process, we compare our model performance with those obtained by solving a CVaR model based on the dynamics of the asset side only. In particular, we maximise the expected return of the portfolio of assets while imposing limits on the CVaR of the losses. The latter are measured as the deviation of the final value of the asset account and the target return under each scenario $l \in \Omega$.

In Figure 1, we show the performances of the selected portfolios. As it can be noted, the optimal portfolio (square) which integrates asset and liability performs better than portfolios solely based on the asset returns (triangle). The latter are obtained by varying the limits on the CVaR of the losses. The sole portfolio which is not dominated by our model corresponds to that with the lowest CVaR. However, observe that no other portfolio yields a lower VaR.

Finally, the optimal portfolio is much more reliable than randomly selected portfolios (diamonds). We here remark that the performances reported in Figure 1 are based on out-of-sample scenarios.

\subsection{The effect of regulatory and equity constraints}

We follow the same procedure seen above to test the impact of regulatory and equity constraints on the out-of-sample performances of the optimal asset-liability portfolios. In Figure 2, we display the performances in terms of VaR and expected ROE for different levels of the minimum guarantee and equity ratio. As expected, high values of $\rho$ (very demanding regulatory requirements) deteriorate the performance of the optimal portfolios. Moreover, such effect is more penalising for policies with high guarantee rates.

We also test the impact of limits on the amount of equity infused by the shareholders. As seen in Section 2.2.2, the equity account monitors the amount of equity to back shortfalls below the minimum guarantee. From an operational point of view, it is important to investigate the performances of the optimal portfolios when the amount of equity is bounded from the above. To this purpose, given a set of scenarios, guarantee rate and equity ratio, we determine the lowest equity bound, $E_{U}^{*}$, such that the model in Equations (18)-(27) delivers a feasible solution. We then solve the same model for increasing levels of the equity upper bound, say $E_{U}=E_{U}^{*}+0.25$. The results are shown in Figure 3. By setting $\rho=4 \%$, we display the performances of the asset-liability portfolios for different $g$ and $E_{U}$. Note that for low levels of the guarantee rate $(g=1 \%)$, the effect of the bound on the equity is negligible. This is because the shortfalls below the minimum guarantee are smaller and, thus, not much equity is needed. As the guarantee rate increases, a marginal change in the equity bound leads to significant changes in the performance of the portfolio. Indeed, for $g=5 \%$, a change of 0.25 unit on the equity bound increases the expected ROE of 0.32 unit and reduces the $\mathrm{VaR}$ of 0.12 unit. Also note that for $g=3 \%$ the marginal gain is smaller and higher levels of the equity bound yield little or no gain. 
Figure 1 The effect of integrating asset and liability. The asset-liability CVaR model shows a superior performance (square) with respect to the asset-only CVaR (triangle) and to naive random portfolios (diamond) (for colours see online version)

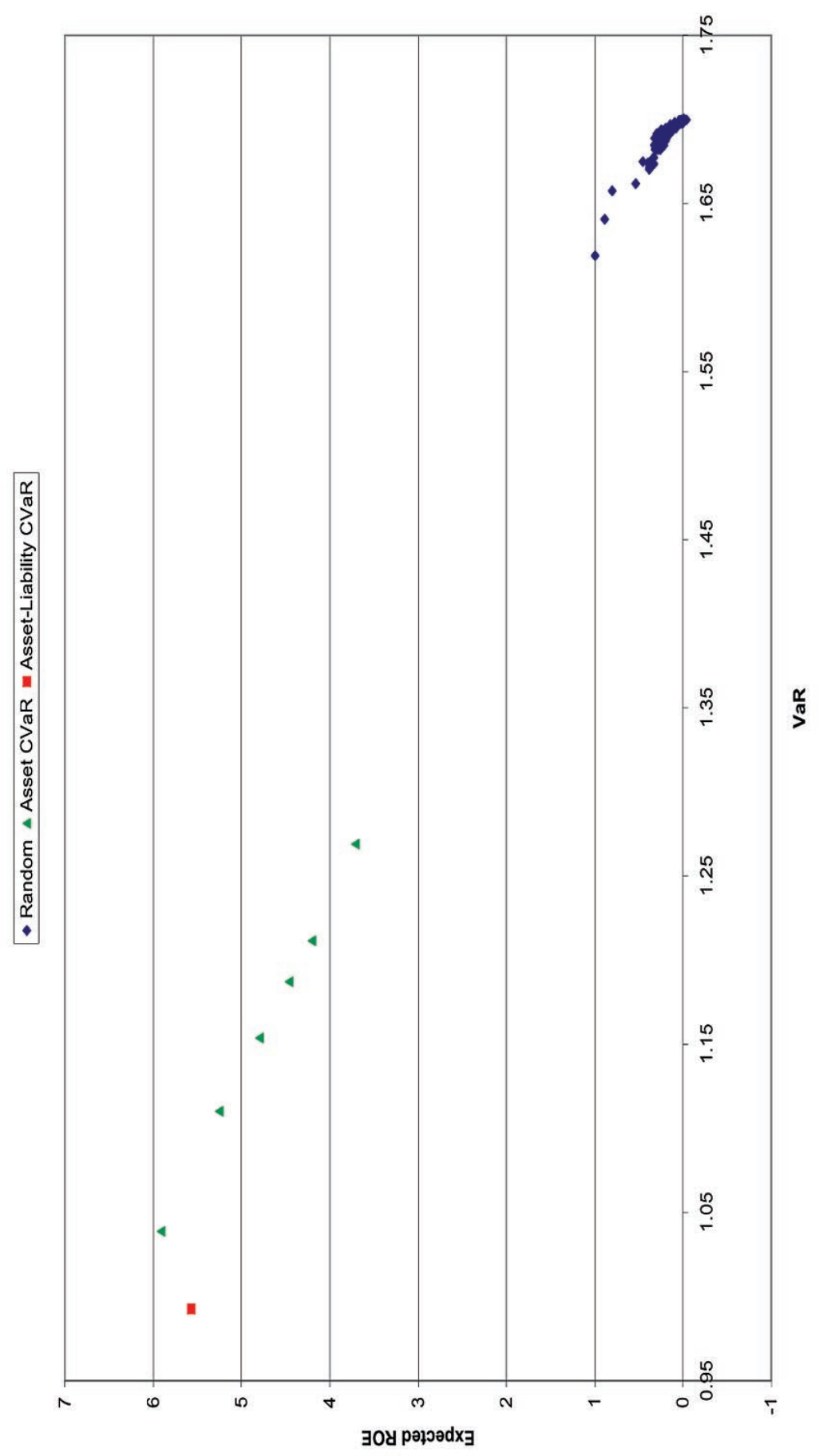


Figure 2 The effect of regulatory constraint. The higher the regulatory parameter, $\rho$, the higher the deterioration of the portfolio performances. Such effect is more penalising for policies with high guarantee rates (for colours see online version)

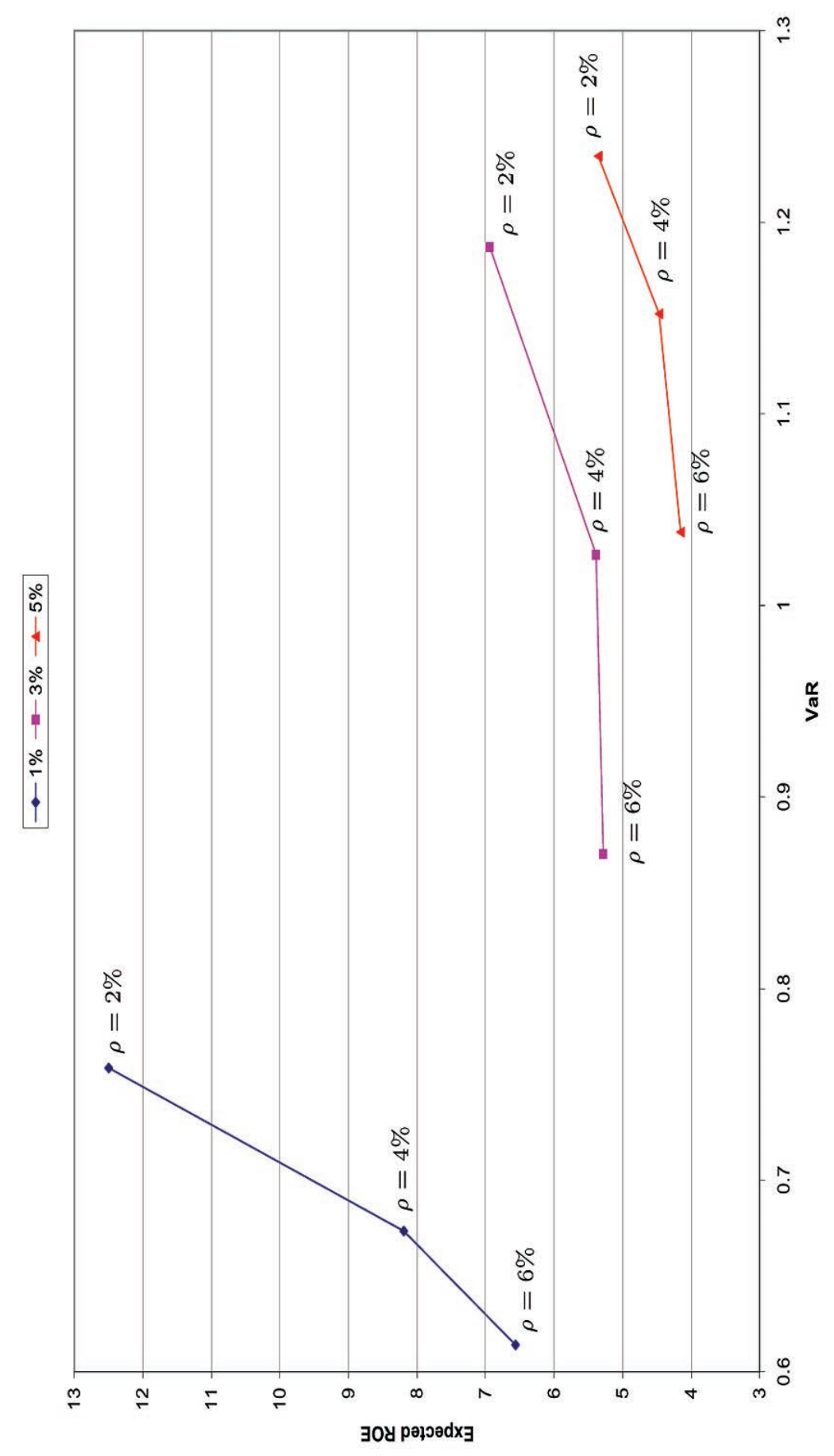


Figure 3 The effect of equity bounds. $E_{U}^{*}$ is the minimum level of equity which yields a feasible solution (for colours see online version)

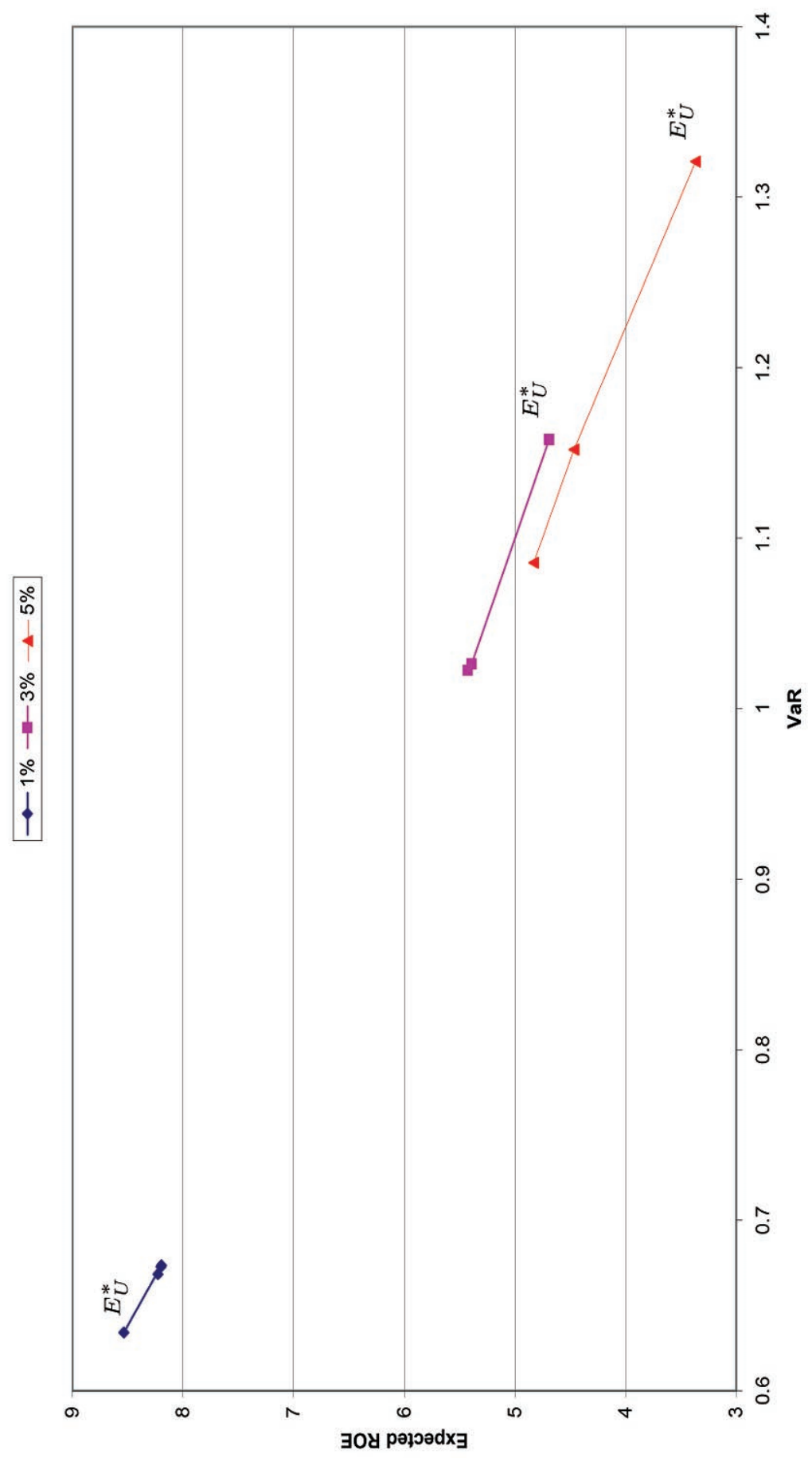


The trade-off shown in Figures 2 and 3 can be used in the design phase of a policy. For example, a very demanding regulatory environment should suggest policies with a lower guarantee rate. This result can also help regulators to understand the impact of their choices on the overall insurance industry and effectively tune their requirements.

\section{Conclusions}

We developed a model to manage insurance products with guarantees. The model is quite general so as to be easily extended to the wider class of products where a baseline return is contractually returned to the investors. The portfolio selected is endogenously determined via a scenario optimisation model. The latter is flexible enough to embed operational and regulatory constraints. We provided empirical results on out-of-sample scenarios and highlighted how the model can be used to design policies and help regulators to set their requirements.

\section{References}

Artzner, P., Delbaen, F., Eber, J. and Heath, D. (1999) 'Coherent measures of risk', Mathematical Finance, Vol. 9, No. 3, pp.203-228.

Bacinello, A.R. (2001) 'Fair pricing of life insurance participating policies with a minimum guarantee', Astin Bulletin, Vol. 31, No. 2, pp.275-297.

Bacinello, A.R. (2003) 'Fair valuation of a guaranteed life insurance participating contract embedding a surrender option', Journal of Risk and Insurance, Vol. 70, No. 3, pp.461-487.

Brooke, A., Kendrick, D. and Meeraus, A. (1992) GAMS: A User's Guide, Release 2.25, Danvers, MA: The Scientific Press, Boyd and Fraser Publishing Company.

Carinõ, D.R. and Ziemba, W.T. (1998) 'Formulation of the Russel-Yasuda Kasai financial planning model', Operations Research, Vol. 46, No. 4, pp.433-449.

Consigli, G. and Dempster, M.A.H. (1998) 'The CALM stochastic programming model for dynamic asset and liability management', in W. Ziemba and J.M. Mulvey (Eds), Worldwide Asset and Liability Modeling, Cambridge, UK: Cambridge University Press, pp.464-500.

Consiglio, A. and Zenios, S.A. (2001) 'Integrated simulation and optimization models for tracking international fixed income indices', Mathematical Programming, Vol. 89, No. 2, pp.311-339.

Consiglio, A., Cocco, F. and Zenios, S.A. (2001) 'The value of integrative risk management for insurance products with minimum guarantees', Journal of Risk Finance, Vol. 2, No. 3, pp.1-11.

Consiglio, A., Cocco, F. and Zenios, S.A. (2007) 'The Prometeia model for managing insurance policies with guarantees', in W.T. Ziemba and S.A. Zenios (Eds), Handbook of Asset and Liability Management 2. Applications and Case Studies, The Netherlands: Elsevier, Handbooks of Finance, Chapter 15, pp.664-705.

Consiglio, A., Cocco, F. and Zenios, S.A. (2008) 'Asset and liability modelling for participating policies with guarantees', European Journal of Operational Research, Vol. 186, No. 1, pp.380-404.

Consiglio, A., Saunders, D. and Zenios, S.A. (2003) 'Insurance league: Italy vs UK', Journal of Risk Finance, Vol. 4, No. 4, pp.47-54. 
Consiglio, A., Saunders, D. and Zenios, S.A. (2006) 'Asset and liability management for insurance products with minimum guarantees: the UK case', Journal of Banking and Finance, Vol. 30, pp.645-667.

Dembo, R. and Mausser, H. (2000) 'The put-call efficient frontier', Algo Research Quarterly, Vol. 3, No. 1, pp.13-25.

Dembo, R. and Rosen, D. (1999) 'The practice of portfolio replication. a practical overview of forward and inverse problems', Annals of Operations Research, Vol. 85, No. 1, pp.267-284.

Dembo, R., Aziz, A., Rosen, D. and Zerbs, M. (2000) Mark To Future - A Framework for Measuring Risk and Reward, Toronto: Algorithmics Publications.

Efron, B. and Tibshirani, R.J. (1998) An Introduction to the Bootstrap, London: Chapman and Hall.

Giraldi, C., Susinno, G., Berti, G., Brunello, J., Buttarazzi, S., Cenciarelli, G., Daroda, C. and Stamegna, G. (2003) 'Insurance optional', in A. Lipton (Ed.), Exotic Options: The Cutting-Edge Collection Technical Papers Published in Risk 1999-2003, Chapter 35, London: Risk Books.

Grosen, A. and Jørgensen, P.L. (2000) 'Fair valuation of life insurance liabilities: the impact of interest rate guarantees, surrender options, and bonus policies', Insurance: Mathematics and Economics, Vol. 26, pp.37-57.

Høyland, K. (1998) 'Asset liability management for a life insurance company: a stochastic programming approach', PhD Thesis, Norwegian University of Science and Technology, Trondheim, Norway.

Kahneman, K. and Tversky, A. (1979) 'Prospect theory: an analysis of decision under risk', Econometrica, Vol. 47, No. 2, pp.263-291.

Miltersen, K.R. and Persson, S. (1999) 'Pricing rate of return guarantees in a Heath-JarrowMorton framework', Insurance: Mathematics and Economics, Vol. 25, pp.307-325.

Mulvey, J.M. and Thorlacius, A.E. (1998) 'The Towers Perrin global capital market scenario generation system', in W. Ziemba and J.M. Mulvey (Eds), Worldwide Asset and Liability Modeling, Cambridge, UK: Cambridge University Press, pp.286-312.

Siglienti, S. (2000) 'Consequences of the reduction of interest rates on insurance', The Geneva Papers on Risk and Insurance, Vol. 25, No. 1, pp.63-77.

Testuri, C.E. and Uryasev, S. (2003) 'On relation between expected regret and conditional value-at-risk', in Z. Rachev (Ed.), Handbook of Numerical Methods in Finance, Boston: Birkhauser.

Uryasev, S. and Rockafellar, R.T. (2000) 'Optimization of conditional value-at-risk', Journal of Risk, Vol. 2, No. 3, pp.21-41.

Wilkie, A.D. (1995) 'More on a stochastic asset model for actuarial use', British Actuarial Journal, Vol. 1, No. 5, pp.777-964.

\section{Note}

1 To simplify the notation, we dropped the functional dependence from the portfolio composition. 